\title{
Veinte años de gobernabilidad y reforma política en Argentina, las causas de la crisis de Diciembre de 2001
}

\author{
Ezequiel Eduardo Parma \\ Universidad de Talca, Talca, Chile. Email: eparma@utalca.cl
}

\begin{abstract}
Resumen: ¿Las sociedades latinoamericanas están mal gobernadas o son simplemente ingobernables? Tal es la incógnita que orienta este trabajo. En el primer caso, los déficits de gobernabilidad que se manifiestan en casi todo el continente serían adjudicables a una insuficiente provisión de gobierno; en el segundo, a una demanda sobredimensionada que superaría toda posibilidad de satisfacción. Este artículo analiza la provisión y demanda de gobierno en un país del Cono Sur, la República Argentina, a lo largo de los últimos 70 años.

Estado.

Palabras clave: Administración pública; políticas públicas; reforma del
\end{abstract}

\section{Twenty years of governance and political reform in Argentina, the causes of the December 2001 crisis}

Abstract: Are Latin American societies poorly governed or are they simply unmanageable? That is the question that guides this work. In the first case, governance deficits that occur in most of the continent would be allocable to an insufficient supply of government; in the second, an oversized demand would exceed any possibility of satisfaction. This paper analyzes the supply and demand of governance in a country of the Southern Cone, Argentina, over the past 70 years.

Key words: Public administration; public policies; State reform.

\section{Vinte anos de governação e reforma política na Argentina, as causas da crise em dezembro de 2001}

Resumo: As sociedades americanas estão mal regulados ou são simplesmente incontroláveis? Essa é a pergunta que orienta este trabalho. No primeiro caso, os déficits de governança que ocorrem na maior parte do continente seria atribuível a um abastecimento insuficiente do governo e, no segundo, a uma demanda de grandes dimensões que excedem qualquer possibilidade de satisfação. Este artigo analisa a oferta ea demanda de governo em um país do Cone Sul, Argentina, nos últimos 70 anos.

Palavras-chave: Administração Pública, Políticas Públicas, Reforma. 


\section{Introducción}

La hipótesis central es que la ingobernabilidad argentina reside en la reiteración de erróneos diagnósticos de situación por parte de los principales actores políticos. En consecuencia, las políticas orientadas a estabilizar las instituciones, desarrollar la economía y distribuir la riqueza, encontraron límites inesperados que les impidieron concretar sus objetivos, llevando a los diferentes gobiernos al fracaso y a la Argentina a la bancarrota.

Los futuros escenarios dependen de que la dirigencia doméstica realice un diagnóstico acertado de la situación nacional, a partir del cual se diseñe un perfil de desarrollo e inserción internacional sustentable.

\section{Diagnóstico}

En 1983, la apertura democrática generó la expectativa de que medio siglo de inestabilidad institucional podría, al fin, ser superado. La primera derrota electoral del peronismo en elecciones limpias, y su aceptación cívica de la derrota, manifestaban la creación embrionaria de un sistema de partidos en el que cada actor relevante aceptaba la victoria del otro sin impugnar la legitimidad del régimen.

En 1989 tendría lugar la primera alternancia democrática de gobierno desde 1916, lo que hizo suponer que la democracia estaba efectivamente consolidada. La prueba final de su fortaleza llegaría el 3 de diciembre de 1990, cuando un amotinamiento militar fue eficazmente reprimido por las fuerzas leales, asegurando la subordinación militar al poder civil.

El periodo que transcurre entre 1983, con la reinstauración de la democracia, y 2001, con el colapso fiscal del estado y la rotación de cinco presidentes en menos de dos semanas, presenta continuidades y rupturas respecto del pasado argentino reciente. La principal ruptura consiste en la estabilidad institucional que se mantiene incólume a pesar de la sucesión de crisis y escándalos militares y económicos. La continuidad, generalmente desatendida por la mayor parte de los observadores contemporáneos, radica en la decadencia de un modelo de acumulación económica que fracasó en transformar la estructura socioproductiva de Argentina de modo sustentable. No obstante las aparentes transformaciones acaecidas durante la década pasada, la declaración del default estatal en diciembre de 2001 desnudó la fragilidad fundamental de la economía argentina.

\section{La persistencia democrática como novedad}

Las transiciones hacia la democracia que tuvieron lugar en América del Sur durante la década de 1980 se desarrollaron en un ambiente de marcado optimismo. Las preocupaciones principales de protagonistas y obser- 
vadores se centraron, en un primer momento, en la consolidación de las instituciones del nuevo régimen.

La amenaza a la democracia seguía visualizándose en las fuerzas armadas, por lo que se consideraba a los pactos políticos entre las fuerzas civiles como el antídoto más efectivo contra un retorno autoritario (Schmitter, O’Donnell y Whitehead 1989).

De esos pactos debían surgir nuevas instituciones que, más flexibles y modernas que en el pasado, dotaran a los gobiernos de las herramientas apropiadas para enfrentar los nuevos desafíos (Linz 1994). Sólo algunos analistas percibieron entonces que la transición en curso era doble, a la vez política y económica (Portantiero, 1987). Las dificultades, como resultaría finalmente claro, superaron la capacidad de los nuevos gobernantes para hacerles frente. En Argentina, semejante desequilibrio quedó de manifiesto cuando el radical Raúl Alfonsín debió renunciar anticipadamente a la presidencia a mediados de 1989, salvando la continuidad democrática pero denotando la debilidad del nuevo gobierno para cabalgar la crisis económica.

La ilusión de que la democracia resolvería todos los problemas se había encarnado en una frase que Alfonsín utilizó frecuentemente durante la campaña electoral: "con democracia se come, con democracia se educa, con democracia se cura”. La asociación con el bienestar, la educación y la salud partían de una concepción de la democracia, más que como un régimen político, como una forma de vida. A diferencia del autoritarismo que se dejaba atrás, la democracia implicaba un compromiso vital con una cultura pluralista y tolerante. El discurso dominante no le demandaba a la democracia que entregara resultados particulares, pues éstos llegarían naturalmente una vez que el sistema estuviera en su lugar.

El espontaneísmo -o simplismo- que informaba semejante discurso se demostraría incapaz de adaptarse a los obstáculos planteados por la crisis del modelo económico que saldría entonces a la luz. Las dos oleadas hiperinflacionarias de 1989 y 1990 marcaron los límites de una estrategia de gobierno que subestimaba el impacto de la economía sobre las expectativas sociales, condicionando fuertemente los márgenes de acción política.

Los diez años que siguieron, signados por el retorno del peronismo al poder y su reinterpretación neoliberal a manos del presidente Carlos Menem, fueron la contracara del gobierno de Alfonsín. Pragmático y populista a un tiempo, Menem desplazó de las prioridades gubernamentales a las reformas institucionales y colocó en el tope de su agenda la cuestión económica y la reforma del estado. De consuno con el clima de época predominante en los 90, su administración llevó a cabo una amplia restructuración que instrumentó los principales puntos del Consenso de Washington: liberalización, apertura, privatización y desregulación.

En pocos años, el estado se retiró de la gestión de importantes sectores económicos como la energía y los servicios públicos, cuya explotación 
fue concedida en su mayoría a empresas extranjeras de origen europeo. Simultáneamente, el gobierno federal restituyó a las provincias la administración de numerosos servicios, entre ellos la educación secundaria, transfiriendo recursos entre niveles del sector público en una maniobra que no redujo el costo fiscal global pero contribuyó a disimularlo.

Las limitaciones de las reformas implementadas por Menem comenzaron a traslucirse a partir de 1995, cuando una serie de shocks externos golpeó duramente a los mercados emergentes. La recesión producida en Argentina puso fin a un lustro de alto crecimiento, dejando además al desnudo las aristas polémicas de la gestión peronista que el éxito económico había disimulado hasta entonces.

La corrupción y frivolidad adjudicadas a Menem y sus funcionarios pasó entonces de característica pintoresca a defecto intolerable. La consecuencia fue la victoria presidencial en 1999 de la Alianza, frente electoral compuesto por el radicalismo y el Frepaso que hacía hincapié en la ética pública pero no avanzaba propuestas concretas de cambio económico.

Dos años después, habiendo dilapidado su capital moral debido a una serie de escándalos y peleas internas y sin haber logrado reactivar la economía, la Alianza abandonaría el gobierno de forma anticipada ante la profundización de la conflictividad social, repitiendo el fracaso radical del decenio anterior. En menos de veinte años, las expectativas optimistas depositadas en la democracia se habían esfumado. El desempeño de tres presidentes electos concluyó en un cuadro de empobrecimiento y desempleo sin parangón en la historia argentina. Sin embargo, el régimen político se mantuvo en funcionamiento y el apoyo popular a la democracia no se redujo de modo significativo (Lagos 2002).

Por primera vez en setenta años, la mala performance de los gobiernos no arrastraba consigo al régimen. La democracia electoral había logrado consolidarse; la gobernancia democrática, en cambio, no.

\section{La decadencia económica como constante}

El respeto a los mecanismos constitucionales de sucesión presidencial no puede ocultar que, en un sistema presidencial definido por el mandato fijo e independiente del jefe de gobierno, la reiteración de recambios presidenciales anticipados constituye una anomalía peligrosa. Esta anomalía, que en un sistema parlamentario podría resultar tolerable, combina estabilidad institucional -si bien irregular — con inestabilidad de gobierno. Semejante combinación es una novedad y, pese a todo, un progreso en la política argentina.

En cambio, el desempeño económico de los gobiernos democráticos se mantuvo dentro de los cánones tradicionales: resultó un fracaso evidente, que implicó el fin de una excepcionalidad histórica. 
Argentina siempre fue un país que no encajaba en su contexto. En un continente racialmente mixto, religiosamente sincrético, educacionalmente pobre y económicamente subdesarrollado, destacarse no era difícil. A lo largo de un siglo y medio, el perfil argentino fue más europeo que latinoamericano. De fisonomía blanca, altos niveles de alfabetización, moderado desarrollo económico y pautas culturales occidentales, sus modelos de referencia siempre fueron París y Londres, no Río de Janeiro o Ciudad de México. La sociedad argentina era integradora y de clase media, a diferencia de las que la circundaban, más pobres y excluyentes.

La excepcionalidad argentina se basó en una estructura económica que manifestó dos etapas claras. Entre la organización nacional en 1880 y el primer golpe de estado en 1930, la producción nacional se centró en el campo y se orientó hacia el mercado mundial. Fue el llamado modelo agroexportador, que se agotó con la crisis mundial de los años 30 . A partir de 1940, los proyectos nacionalistas perfilaron una producción basada en la industria y orientada hacia el mercado interno. Se conoció como el modelo de industrialización por sustitución de importaciones, sostenido en esa época por la Comisión Económica para América Latina (CEPAL) de las Naciones Unidas.

Este segundo modelo nació limitado, en un mundo que se reconstruía velozmente después de la guerra, y se agotó mucho antes de lograr sus objetivos autárquicos y desarrollistas. A partir de 1970, Argentina se encontró estancada y sin un rumbo claro de desarrollo, sin lograr definir qué producir y a quién vendérselo. A la ausencia de un modelo de acumulación se le agregó una práctica perversa: la de gastar más de lo que se produce. Así, a partir de 1946 los sucesivos gobiernos financiaron los permanentes déficits públicos mediante cuatro mecanismos centrales: la apropiación de los fondos públicos de pensión, la inflación, la liquidación de los activos estatales (privatizaciones) y el endeudamiento extremo.

El año 1991 constituye un punto de inflexión, en cuanto la vieja política estado-céntrica sostenida sobre recursos inflacionarios dio lugar a una política neoliberal, que eliminaba la inflación para financiarse por medio de las privatizaciones y de un mayor endeudamiento público. No obstante el cambio en las fuentes de financiamiento estatal, la constante siguió siendo el déficit fiscal y la baja eficiencia del estado.

Finalmente, el dinero se acabó. En 2001, al estado argentino se le cerraron las últimas fuentes de recursos (los préstamos externos) y debió declarar la cesación de pagos de su deuda. Los argentinos descubren ahora la realidad de que su estándar de vida estuvo demasiado tiempo por encima de sus posibilidades reales, y ahora deben pagar las cuentas por lo gastado a crédito. En otros términos, la bancarrota significa ajuste. Y no es fácil convertirse en pobre habiendo sido rico toda la vida -o, más bien, habiendo creído ser rico. En 1920, el PBI per capita argentino representaba más del 80\% del de los Estados Unidos. En 2000, antes del colapso, no llegaba al 40\% (Prados de la Escosura 2000). 
En 2002 probablemente se encuentre por debajo del 10\%. Semejante caída en la capacidad económica de una sociedad no puede sino impactar sobre las expectativas sociales, con consecuencias negativas sobre la legitimidad del orden político al que se responsabiliza por la situación. En las elecciones de octubre de 2001 la ciudadanía envío un mensaje que ni el gobierno ni la oposición supieron traducir en decisiones públicas. Por primera vez se manifestó, en esas elecciones, un rechazo generalizado contra la dirigencia política, expresado a través de un masivo aumento del voto intencionalmente anulado. El voto en blanco y la abstención electoral también crecieron, pero no alcanzaron tanta magnitud como el voto nulo. En 2002, el repudio ciudadano a la política y sus representantes alcanza niveles extremadamente altos, y ni siquiera los pocos dirigentes bien considerados por el público superan el $40 \%$ de imagen positiva en un marco de fragmentación partidaria inédito. Surge entonces un dilema: ¿cómo gobernar una sociedad que desconfía y rechaza tan drásticamente a todos los que pretenden representarla?

\section{Causas y perspectivas}

Entre 1960 y 1980, varios autores intentaron explicar las razones de la inestabilidad política argentina. Los principales ensayos interpretativos pueden ser agrupados en torno a tres ejes de análisis (De Luca y Malamud 1996): el “empate social” (Portantiero 1973; O’Donnell 1972, 1977), la “crisis de legitimidad” (Botana 1988; Mora y Araujo 1978, 1982) y la "ausencia de un partido de derecha” (Di Tella 1971/72; Mora y Araujo 1982). Una somera revisión de cada enfoque es conveniente para mejor comprender los cambios acaecidos desde 1983.

\section{El debate sobre la crisis crónica}

El "empate social” constituyó una situación caracterizada por una relación de fuerzas sociales "alternativamente capaces de vetar los proyectos de las otras, pero sin recursos suficientes para imponer, de manera perdurable, los propios” (Portantiero, 1987a: 76). Para O’Donnell, la lógica particular de desarrollo del capitalismo en Argentina llevaba a una situación donde dos grandes bloques sociales se disputaban la dirección del proceso económico, mientras un tercer actor pendulaba cíclicamente para consagrar al vencedor de circunstancia (O’Donnell, 1977). Pese a ello, en ningún caso la victoria podía ser considerada definitiva, ya que la inercia del péndulo volvería a cambiar la fortuna de los participantes. Sólo quedaba, entonces, la posibilidad de impugnar el resultado desfavorable a través del bloqueo mutuo, en una situación en que nadie podía imponer al otro una pauta de dominación permanente.

Para Natalio Botana, en cambio, la inestabilidad política nacional 
es resultado de una crisis de legitimidad, es decir, de un desacuerdo de los actores políticos respecto a las reglas de sucesión en el poder y de una falta de creencias compartidas por gobernantes y gobernados sobre los fines últimos del gobierno y de la sociedad (Botana 1988). Para explicar la ausencia de un proyecto de "sociedad deseable" compartido por gobernantes y gobernados, Botana también avanza la hipótesis de la coexistencia de "tradiciones políticas antagónicas”.

Por su parte, para Manuel Mora y Araujo (Mora y Araujo 1982), la hipótesis de una “crisis de legitimidad” también proporciona la base adecuada de un marco analítico alternativo a otros paradigmas sobre la inestabilidad política argentina. Este autor plantea la coexistencia de dos criterios de legitimidad democrática que pugnan por consolidarse. Por un lado, la idea de una democracia "pluralista/competitiva” inspirada en el sistema representativo, los partidos políticos, la asociación libre y el gobierno limitado. Por el otro, el principio de una democracia “mayoritaria/plebiscitaria”, basado en el respaldo de una mayoría electoral y en el gobierno sin restricciones formales.

Mora y Araujo (1978, 1982) realiza una síntesis eficaz de los enfoques anteriores y le agrega la componente del partido ausente. A partir de su concepción de la "crisis de legitimidad" como enraizada en la estructura social, él argumenta que la viabilidad de un régimen político abierto estaría librada a la conformación y éxito electoral de una fuerza política “de derecha”, capaz de reforzar a una coalición social con pretenciones de resolver el “empate”. En estas condiciones, a las que el autor consideraba viables, se aseguraría una efectiva estabilidad del sistema político. Torcuato Di Tella sostenía a principios de los 70 una hipótesis similar, pero sus conclusiones eran menos optimistas: según su criterio, "lo que ocurre es que en la Argentina los problemas son tan complejos que, simplemente, no se los puede resolver” (Di Tella, 1971/72: 317, destacado en el original).

Más allá de que la contundencia de esta expresión se relativizaba posteriormente con el agregado de "en menos de treinta o cuarenta años", prudente plazo dentro del cual Argentina todavía estaría inmersa, su axioma encuentra algún eco en el argumento de O’Donnell plasmado en "El juego imposible” (O’Donnell 1972) y en determinadas descripciones de Mora y Araujo (Mora y Araujo 1982).

La restauración y persistencia democrática, fenómeno original en medio siglo de historia argentina, permitió que cada una de estas variantes explicativas argumentara los cambios girando alrededor de su propia concepción. Los enfoques del “empate social” experimentaron una evolución desde la unicausalidad -y cierto determinismo económico- hacia aspectos más institucionales y perspectivas analíticas más amplias. Los análisis que se centraban en la "crisis de legitimidad” fundamentan que el consenso post-dictatorial, generado a partir de los abusos represivos del gobierno militar y de la derrota de Malvinas, creó las condiciones para que los actores políticos del campo civil encontraran mínimos comunes denominado- 
res en el juego democrático. Por su parte, las hipótesis del "partido de derecha ausente” surgidas desde una matriz analítica del equilibrio político, encontraron en la transformación del peronismo por Carlos Menem el partido que les aseguraba la defensa de sus intereses.

En 2002, el régimen democrático no aparece en peligro (Lagos 2002). Y sin embargo, la inestabilidad argentina persiste. La diferencia con el pasado es que ahora la inestabilidad se ha transferido desde el régimen hacia el gobierno, y lo que genera no son rupturas democráticas -golpes-sino interrupciones en el calendario constitucional -ingobernabilidad. Conviene entonces ampliar el enfoque, a fin de explicar los motivos estructurales e institucionales del colapso argentino.

\section{La naturaleza de la crisis actual}

La crisis que enfrenta Argentina a inicios del siglo XXI es simultáneamente política y económica. Pocos antecedentes históricos revisten semejante complejidad. La crisis política se asemeja, quizás, a la de los últimos años de la IV República Francesa, cuando las instituciones políticas agotaron su legitimidad preanunciando un cambio de régimen. La crisis económica se asemeja, por su gravedad, a la Gran Depresión americana de los años 30 -si bien las causas son diferentes.

La diferencia radica en que tanto Francia como Estados Unidos enfrentaron una sola crisis, política o económica, a la vez. En América Latina, el caso más parecido es el venezolano, en el que una élite dirigente corrupta e incompetente despilfarró una enorme riqueza natural sin desarrollar la economía nacional y contribuyendo al colapso de las instituciones tradicionales que culminó en el ascenso al poder de Hugo Chávez.

Las crisis política y económica argentinas pueden, cada una, desdoblarse en dos. Por un lado, la crisis política expresa un déficit de legitimidad tanto como una profunda ineficiencia administrativa. Por el otro, la crisis económica implica un grave déficit fiscal junto con serios problemas de productividad. La legitimidad es el problema más visible del sistema político actual, pero es razonable afirmar que ese problema deriva del secular mal funcionamiento -subadministración- del estado, más que de una impugnación a los principios republicanos. En cuanto a la economía, es tan serio el bajísimo crecimiento de la productividad en las últimas tres décadas como la permanente insolvencia fiscal del estado, cuyo déficit absorbe recursos del mercado limitando el crédito para la inversión e innovación privada, y sin proveer siquiera una eficaz orientación o regulación pública.

Si bien la situación argentina se ha descrito hasta acá como una crisis múltiple, producto de la convergencia y potenciación de varios factores, el escenario resultante será aún más complejo. El motivo es que esta crisis no es una expresión de problemas transitorios, sino la manifestación más 
acabada de una monumental implosión social, política y económica. Cuando se disipen las brumas de la confusión aún reinante, Argentina se encontrará en un estado de decadencia generalizada que será difícilmente asimilable por su población.

Según cálculos optimistas, las condiciones deberían estabilizarse en el próximo año y la economía crecer durante una década a altas tasas para recuperar los niveles de 1998. ¿Cuáles son las razones por las que se produjo la debacle, y cuáles los instrumentos que podrían colaborar para superarla?

Según resulta expresado en todas las encuestas de opinión, la ciudadanía argentina considera la corrupción de la dirigencia como principal culpable de la situación. El respaldo público a las instituciones y personalidades de gobierno es extremadamente reducido, y el capital social del país aparece disuelto. Los niveles de reputación y confianza de los líderes alcanzan los mínimos históricos, y al fenómeno del voto nulo se suman las manifestaciones públicas llamadas cacerolazos, mayoritariamente compuestas por las clases medias, y piquetes, por las clases bajas. La demanda más sentida es "que se vayan todos", en referencia a los dirigentes de todos los partidos y sectores a los que se responsabiliza por la situación.

Pero, pese al colapso y las protestas, no han surgido alternativas de política ni liderazgos renovados que encarnen una posibilidad de cambio. La crisis parece enrollarse sobre sí misma, sin horizonte de salida. Es precisamente la cuestión de los horizontes la que orienta la bibliografía que, en los últimos años, ha pretendido dar una explicación a la reiteración cíclica de los problemas argentinas. En esta veta, Spiller y Tommasi (2000) presentan una agenda de puntos críticos y una agenda de reformas institucionales.

En su diagnóstico, las causas de la decadencia argentina son fundamentalmente institucionales. El inconveniente central del sistema político argentino reside en que su estructura de incentivos "ha propiciado la creación de políticas erráticas y la dificultad de generar acuerdos intertemporales que sustenten la generación de políticas eficientes de largo plazo” (Spiller y Tommasi 2000: 456). Por lo tanto, la solución consiste en modificar las instituciones de gobierno, en especial el Congreso y la estructura del federalismo fiscal pero también la burocracia, la presidencia y el Poder Judicial, para generar beneficios a los actores que orienten sus decisiones considerando plazos medios y largos.

Los enfoques que interpretan la crisis a la luz de la nueva economía institucional no son privativos de Argentina, sino que su aplicación se está extendiendo rápidamente a toda América Latina. Así, en una revisión comparada de siete obras sobre reforma en el subcontinente que evalúan el impacto diferencial de las fallas de mercado y las fallas de estado, Starr (2002) concluye que el fracaso de las reformas económicas de los 90 reconoce causas políticas e institucionales. Por lo tanto, eficaces políticas 
regulatorias y de competencia sólo serán correctamente implementadas cuando se haya extendido el horizonte temporal de los decisores políticos a partir de una reforma de las instituciones políticas.

Algunos analistas, sin embargo, destacan que una reforma política que se centre sólo en las instituciones de representación -es decir, en la estructura de toma de decisiones - resultaría incompleta. En su visión, el problema radica no sólo en la formulación de políticas, sino en su implementación e impacto (Acuña 2002). Por ello, la reforma del estado es un complemento indispensable de cualquier estrategia reformista. De algún modo, la recuperación de este nivel de análisis refleja la inquietud que O’Donnell (1993) manifestara desde mediados de la década pasada, en el sentido que un enfoque limitado al régimen político no capta las deficiencias producidas en el sistema político por el funcionamiento errático del estado.

\section{Conclusiones parciales acerca de crisis y reformas}

A lo largo de este trabajo se ha procurado describir la situación argentina actual, a fin de explicar lo que ésta tiene de original y lo que expresa como continuidad histórica. Seguidamente se han enumerado las razones que la literatura ofrece para comprender tal situación, y mencionado algunos de los ejes que deben informar cualquier reflexión sobre la superación de la crisis. En esta parte se resumen los argumentos y se analizan las opciones que contribuirían a diseñar un proyecto sustentable de reforma política -de la estructura de gobernancia.

La crisis argentina es hoy percibida, según se expresa en los principales medios de comunicación y en los relevamientos de opinión pública, como una crisis de representatividad. Así, se argumenta que el divorcio entre la ciudadanía y los representantes -manifestado en la alta volatilidad electoral, el abstencionismo y el voto voluntariamente anulado- debería ser combatido mediante una reforma política que acerque a los representantes al "pueblo". Esta demanda de cambio se centra en el sistema electoral -al que se propone convertir en uni o binominal_- la financiación partidaria y los mecanismos de selección de candidatos.

La dirigencia política establecida, por su parte, ha reaccionado defensivamente contra las demandas de cambio, promoviendo reformas menores o parciales y rechazando la generalización implícita tras el clamor "que se vayan todos”. Esta reacción de la dirigencia, que no ha querido o no ha logrado acuerdos amplios de reforma, retroalimenta a su vez la insatisfacción ciudadana, cuyo nivel de aceptación de las opciones y líderes políticos se encuentra consistentemente en su piso histórico.

Este trabajo ha argumentado que la secuencia recién descripta (divorcio representantes/representados - demanda de cambio -respuesta in- 
suficiente- aumento del divorcio) contribuye a ocultar dos cuestiones relevantes. Por un lado, no se entiende acabadamente que la reforma política, si no es acompañada por una reforma del estado, resultará insuficiente y frustrará las expectativas de sus mismos mentores.

Por el otro, no se percibe que la crisis argentina no es una etapa de cambio sino de decadencia, y que la superación de la crisis dejará al descubierto un país mucho más pobre, más injusto y más violento que antes. Esta abrupta latinoamericanización de Argentina probablemente aumentará la frustración de sus habitantes, cuya autopercepción sobrestima los medios reales con que contará en el futuro cercano.

Desde el inicio de las transiciones democráticas en América Latina, a partir de 1980, la cuestión del tipo de régimen (democracia o dictadura, presidencialismo o parlamentarismo) constituyó la base del pensamiento de observadores y protagonistas políticos. Asumida la democracia, en los 90 el foco se centró en los procesos de liberalización económica, que apuntaron a la privatización, desregulación y ajuste estructural. El fracaso de estas políticas en casi todo el continente es hoy evidente, y se teme que arrastre también a la democracia.

Los liderazgos neopopulistas, que combinaron caudillismo tradicional con vocación reformista neoliberal, fueron la clave para explicar los procesos de cambio de los 90 . El argumento usual es que este fenómeno, también denominado democracia delegativa, habría ido erosionando las instituciones del régimen hasta convertirlo en una fachada sin demasiado contenido democrático real. Sin embargo, y desde una perspectiva comparada, la amenaza que enfrenta hoy América Latina no es la quiebra de la democracia sino el colapso del estado. Si el régimen político es el conjunto de reglas que establece quién toma las decisiones y de qué manera, el estado es el aparato que permite la implementación de tales decisiones. A inicios del nuevo siglo, la crisis fiscal y la dificultad para garantizar el orden público se constituyen en la clave que explica el fracaso de nuestros países.

Cualquier decisión que se tome, buena o mala, progresista o conservadora, es inútil sin implementación. La reconstrucción del aparato del estado, que implica un funcionariado estable, competente e independiente de los vaivenes partidarios, es condición sine qua non para la recomposición social en América Latina. Y ello exige una capacidad recaudatoria que los estados de la región, con relativas excepciones, no han logrado construir.

Si los sistemas representativos modernos surgieron a partir de la demanda "no taxation without representation", ¿es concebible la mantención de sistemas representativos allí dónde la ilegitimidad del estado conlleva a la ciudadanía a actuar como si el lema fuera, simplemente, "no taxation"? Construir estados es un proceso lento; destruirlos, está visto, lleva mucho menos tiempo. 
En todo sistema político existe un trade off entre representatividad (abarcatividad y legitimidad de la representación) y eficacia operativa (capacidad de formular e implementar políticas públicas). La persistencia de fuertes identificaciones partidarias no obstante la prolongada decadencia argentina indica que no es la representatividad la pata renga de la democracia argentina, sino la eficacia.

Paradojalmente, sin embargo, gran parte de las propuestas enarboladas por los políticos argentinos que se plantean como alternativa al duopolio radical-peronista se enanca sobre la "crisis de representatividad" en vez de sobre la ingobernabilidad permanente. Si las conclusiones que acá se infieren son correctas, la legitimidad -0, en otras palabras, la duración- del sistema político argentino dependerá más de los resultados (outcomes) que de los principios de representación. Y los resultados dependerán a su vez de la estructura de incentivos, o estructura de gobernancia, en función de la extensión del horizonte temporal que oriente a los actores.

¿Pero quiénes serán los actores con los incentivos suficientes para modificar la estructura de gobernancia? Weber habría respondido que son los líderes carismáticos los que promueven el cambio institucional; la experiencia argentina alerta contra esta respuesta y alienta, quizás, una estrategia menos riesgosa - pero más fatigosa - de cooperación colectiva. La ecuación plausible requeriría de instituciones políticas que aseguren transparencia y horizontes de largo plazo, de un estado que goce de autonomía y eficacia y de un mercado que garantice a la vez regulación y competencia. Los pronósticos no pueden ser demasiado optimistas. 


\section{Bibliografía}

Acuña, Carlos (2002), Conferencia sobre Reforma Política. Universidad Torcuato Di Tella, mayo, Buenos Aires.

Botana, Natalio (1988), "La tradición política en la Argentina moderna.” Pinto, Julio (comp.), Ensayos sobre la crisis política argentina. Centro Editor de América Latina-CEAL, Biblioteca Política Argentina No 206, Buenos Aires.

De Luca, Miguel y Andrés Malamud (1996), “La estabilidad democrática en la Argentina de fin de siglo.” Julio Pinto (comp.), Las nuevas democracias del Cono Sur: cambios y continuidades. Oficina de Publicaciones del CBC, Universidad de Buenos Aires.

Di Tella, Torcuato (1971/2), “La búsqueda de la fórmula política argentina.” Desarrollo Económico, N 42/44 Vol. 1, Buenos Aires.

Lagos, Marta (2002), Latinobarómetro 1996-2002. Datos preliminares 2002. Taller Primer Congreso Latinoamericano de Ciencia Política, Salamanca.

LeDuc, Lawrence (1996), “Elections and democratic governance”. Lawrence LeDuc, Richard G. Niemi and Pippa Norris, eds., Comparing Democracies. Elections and Voting in Global Perspective. Thousand Oaks, Sage.

Linz, Juan J. (1994), Presidential or Parliamentary Democracy: Does It Make a Difference?

Linz, Juan J. y Valenzuela, Arturo, The failure of presidential democracy. Johns Hopkins University Press, Baltimore.

Mora y Araujo, Manuel (1978), "Procesos electorales y fuerzas políticas. Una perspectiva analítica.” Beltrán, Virgilio (ed.), El futuro político de la Argentina. ITDT, Buenos Aires.

Ídem (1982), “El ciclo político argentino.” Desarrollo Económico № 86 Vol. 22, Buenos Aires.

O’Donnell, Guillermo (1972), Modernización y autoritarismo. Paidós, Buenos Aires.

Ídem (1977), “Estado y alianzas en la Argentina, 1956-1976.” Desarrollo Económico $\mathrm{N}^{\circ} 64$ Vol. 16, Buenos Aires.

Ídem (1993), “Acerca del estado, la democratización y algunos problemas conceptuales. Una perspectiva latinoamericana con referencias a países poscomunistas.” Desarrollo Eonómico No 130 Vol. 33: Buenos Aires. 
Portantiero, Juan Carlos (1973), “Clases dominantes y crisis política en la Argentina.” Oscar Braun (comp.), El capitalismo argentino en crisis. Siglo XXI, Buenos Aires.

Ídem (1987), “La transición entre la confrontación y el acuerdo.” Nun, José y Portantiero, Juan Carlos (comps.), Ensayos sobre la transición democrática en la Argentina. Puntosur, Buenos Aires.

Prados de la Escosura (2000), “International Comparisons of Real Product, 1820-1990.” Explorations in Economic History No 37: 1-41.

Spiller, Pablo T. y Mariano Tommasi (2000), "El funcionamiento de las instituciones políticas y las políticas públicas en la Argentina: una aproximación desde la nueva economía institucional”. Desarrollo Económico, Vol. $40 \mathrm{~N}^{\circ} 159$.

Schmitter, Philippe, Guillermo O’Donnell y Lawrence Whitehead (1989), Transiciones desde un gobierno autoritario. Paidós, Buenos Aires (4 volúmenes).

Starr, Pamela K. (2002), "Perfecting Reform in Latin America: What Role for the State?” Latin America Research Review, Vol 37 Nº 2.

Recibido: 03.08.2012

Aceptado: 16.01.2013 